\title{
Comparative Analyses of Historical Trends in Confirmed Dengue Illnesses Detected at Public Hospitals in Bangkok and Northern Thailand, 2002-2018
}

\author{
Phirangkul Kerdpanich, ${ }^{1}$ Suthinee Kongkiatngam, ${ }^{2}$ Darunee Buddhari, ${ }^{3}$ Sriluck Simasathien, ${ }^{1}$ Chonticha Klungthong, ${ }^{4}$ \\ Prinyada Rodpradit, ${ }^{4}$ Butsaya Thaisomboonsuk, ${ }^{4}$ Tippa Wongstitwilairoong, ${ }^{4}$ Taweewun Hunsawong, ${ }^{4}$ Kathryn B. Anderson, ${ }^{4,5,6,7}$ \\ Stefan Fernandez, ${ }^{4}$ and Anthony R. Jones ${ }^{4 *}$ \\ ${ }^{1}$ Department of Pediatrics, Phramongkutklao Hospital, Bangkok, Thailand; ${ }^{2}$ Department of Pediatrics, Kamphaeng Phet Provincial Hospital \\ (KPPH), Kamphaeng Phet, Thailand; ${ }^{3}$ Kamphaeng Phet-AFRIMS Virology Research Unit, AFRIMS, Kamphaeng Phet, Thailand; ${ }^{4}$ Department of \\ Virology, Armed Forces Research Institute of Medical Sciences (AFRIMS), Bangkok, Thailand; ${ }^{5}$ Department of Medicine, SUNY Upstate Medical \\ University, Syracuse, New York; ${ }^{6}$ Department of Microbiology and Immunology, SUNY Upstate Medical University, Syracuse, New York; ${ }^{7}$ Institute \\ for Global Health and Translational Sciences, SUNY Upstate Medical University, Syracuse, New York
}

\begin{abstract}
Dengue is a re-emerging global public health problem, the most common arbovirus causing human disease in the world, and a major cause of hospitalization in endemic countries causing significant economic burden. Data were analyzed from passive surveillance of hospital-attended dengue cases from 2002 to 2018 at Phramongkutklao Hospital (PMKH) located in Bangkok, Thailand, and Kamphaeng Phet Provincial Hospital (KPPH) located in the lower northern region of Thailand. At PMKH, serotype 1 proved to be the most common strain of the virus, whereas at KPPH, serotypes 1 , 2, and 3 were the most common strains from 2006 to 2008, 2009 to 2012, and 2013 to 2015, respectively. The 11-17 years age-group made up the largest proportion of patients impacted by dengue illnesses during the study period at both sites. At KPPH, dengue virus (DENV)-3 was responsible for most cases of dengue fever (DF), whereas it was DENV-1 at PMKH. In cases where dengue hemorrhagic fever was the clinical diagnosis, DENV-2 was the predominant serotype at KPPH, whereas at PMKH, it was DENV-1. The overall disease prevalence remained consistent across the two study sites with DF being the predominant clinical diagnosis as the result of an acute secondary dengue infection, representing $40.7 \%$ of overall cases at KPPH and 56.8\% at PMKH. The differences seen between these sites could be a result of climate change increasing the length of dengue season and shifts in migration patterns of these populations from rural to urban areas and vice versa.
\end{abstract}

\section{INTRODUCTION}

Dengue virus (DENV) is an important arboviral pathogen causing febrile illness and its more serious manifestation, dengue hemorrhagic fever (DHF)/dengue shock syndrome (DSS). Dengue is a re-emerging global public health problem, a major cause of hospitalization in endemic countries, causes significant economic burden, and is the most common arbovirus causing human disease in the world, with an estimated 3.9 billion people in 128 countries at risk of infection with DENVs. ${ }^{1,2}$ Dengue is a public health priority in Southeast Asia, and Thailand contributes substantially to the regional disease burden. From 2000 to 2011 variations in incidences occurred, with epidemics in 2001, 2008, and 2010 all the while remaining a highly seasonal disease as well. In addition, age-group distribution of dengue disease shifted from younger toward older persons, and heterogeneous geographical patterns of the disease were observed, including higher incidence rates reported in the South of Thailand with serotype distribution variations introduced with respect to both time and place. ${ }^{3}$

Seasonal endemic transmission can lead to epidemics in susceptible populations. Previous studies conducted by the Armed Forces Research Institute of Medical Sciences (AFRIMS) in Kamphaeng Phet, Thailand, a well-established dengue-endemic area of approximately 700,000 people in a mostly rural to semi-rural setting, have described: the spatial and temporal dynamics of DENV transmission, the incidence of DENV infection and risk factors for severe disease, and the full burden of DENV infections with the risk factors specific to

\footnotetext{
${ }^{*}$ Address correspondence to Anthony R. Jones, Department of Virology, Armed Forces Research Institute of Medical Sciences (AFRIMS), 315/6 Ratchawithi Rd., Thung Phaya Thai, Ratchathewi,
} Bangkok 10400, Thailand. E-mail: anthony.jones.mil@afrims.org each. $^{2,4-8}$ This semi-rural setting differs from that of Phramongkutklao Hospital (PMKH), a military hospital located in urban Bangkok that serves both civilians and members of the Royal Thai Army. ${ }^{9-11}$

This study served to elucidate if there is a difference in dengue cases and clinical severity based on the location of the patient, whether they are seen at PMKH or Kamphaeng Phet Provincial Hospital (KPPH) (Figure 1). This was accomplished through several means to include 1) the characterization and comparison of the epidemiology of DENV infections as detected among individuals presenting for inpatient care at $\mathrm{PMKH}$ (Bangkok) and inpatient or outpatient care at KPPH (Kamphaeng Phet) from 2002 to 2018, 2) through the comparison of the temporal features of DENV transmission in Bangkok and Kamphaeng Phet, Thailand, including DENV serotype, ages of infection, primary/secondary infection, as well as other important demographic variables, 3) the description and comparison of the features of DENV infection in Bangkok and Kamphaeng Phet with respect to the occurrence of severe dengue by demographic predictors and DENV serotype, 4) and the impact the varying population pools have on dengue detection assays to include polymerase chain reaction.

\section{METHODS}

Ethics statement. The retrieval and analysis of coded preexisting data in this study was approved by the Institutional Review Boards of Walter Reed Army Institute of Research, Royal Thai Army, and the Institute for the Development of Human Research Protections Thai Ministry of Public Health, IRB RTA 679/2563. All data were analyzed anonymously.

Description of the dataset. The analyzed data were from public health samples collected during passive surveillance of 

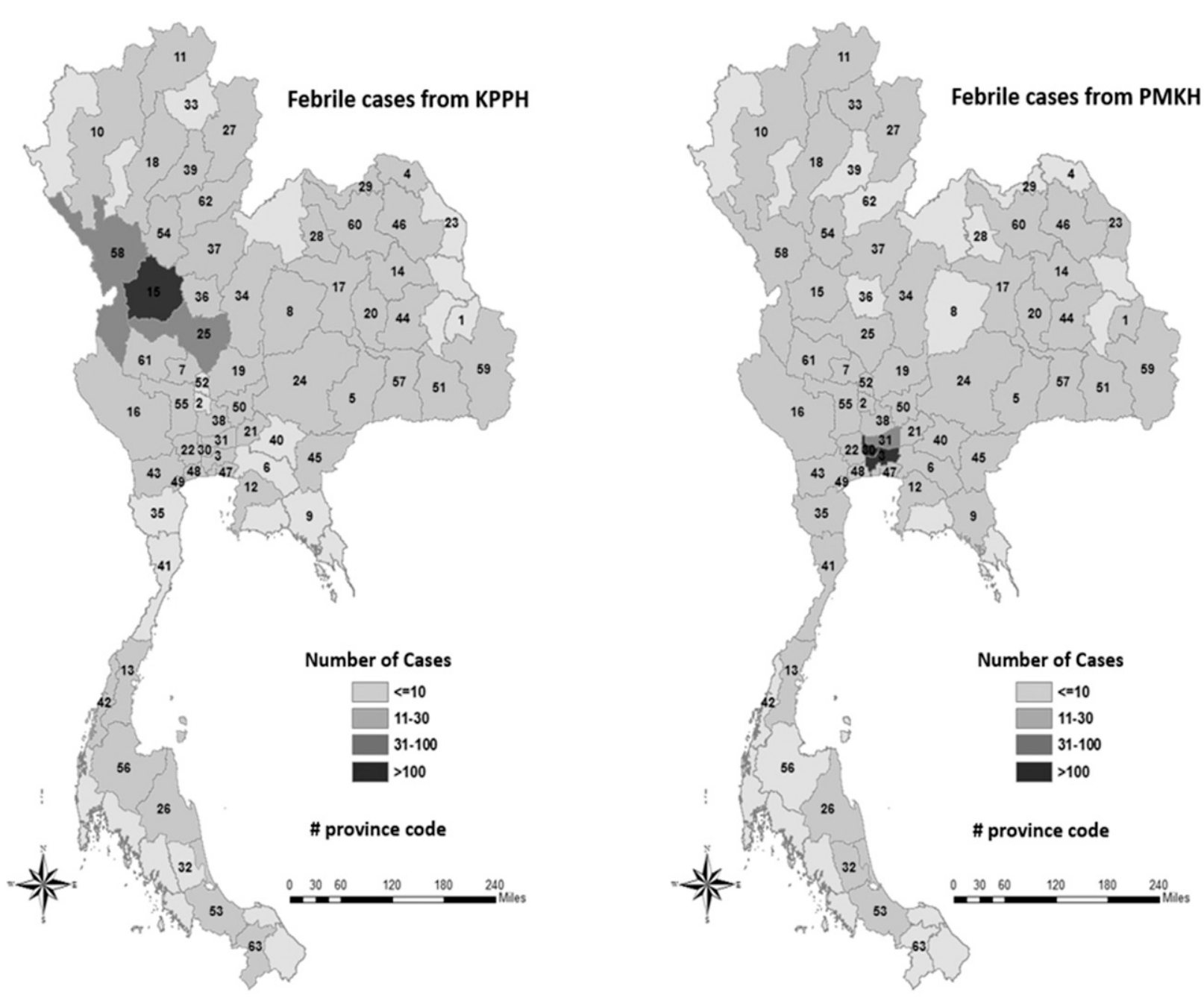

\begin{tabular}{|c|c|c|c|c|}
\hline 1. Amnat Charoen & 14. Kalasin & 27. Nan & 40. Prachin Buri & 53. Songkhla \\
\hline 2. Ang Thong & 15. Kamphaeng Phet & 28. Nong Bua Lam Phu & 41. Prachuap Khiri Khan & 54. Sukhothai \\
\hline 3. Bangkok Metropolis & 16. Kanchanaburi & 29. Nong Khai & 42. Ranong & 55. Suphan Buri \\
\hline 4. Bueng Kan & 17. Khon Kaen & 30. Nonthaburi & 43. Ratchaburi & 56. Surat Thani \\
\hline 5. Buri Ram & 18. Lampang & 31. Pathum Thani & 44. Roi Et & 57. Surin \\
\hline 6. Chachoengsao & 19. Lop Buri & 32. Phatthalung & 45. Sa Kaeo & 58. Tak \\
\hline 7. Chai Nat & 20. Maha Sarakham & 33. Phayao & 46. Sakon Nakhon & 59. Ubon Ratchathani \\
\hline 8. Chaiyaphum & 21. Nakhon Nayok & 34. Phetchabun & 47. Samut Prakan & 60. Udon Thani \\
\hline 9. Chanthaburi & 22. Nakhon Pathom & 35. Phetchaburi & 48. Samut Sakhon & 61. Uthai Thani \\
\hline 10. Chiang Mai & 23. Nakhon Phanom & 36. Phichit & 49. Samut Songkhram & 62. Uttaradit \\
\hline 11. Chiang Rai & 24. Nakhon Ratchasima & 37. Phitsanulok & 50. Saraburi & 63. Yala \\
\hline 12. Chon Buri & 25. Nakhon Sawan & 38. Phra Nakhon Si Ayutthaya & 51. Si Sa Ket & \\
\hline 13. Chumphon & 26. Nakhon Si Thammarat & 39. Phrae & 52. Sing Buri & \\
\hline
\end{tabular}

FIGURE 1. Heat map displaying the febrile cases in residence from Kamphaeng Phet Provincial Hospital (KPPH) and Phramongkutklao Hospital (PMKH) during the study period (2002-2018).

hospital-attended dengue cases from 2002 to 2018 at PMKH, a 1,200-bed tertiary care military hospital located in Bangkok capital of Thailand, and KPPH, a 410-bed provincial civilian hospital located in lower northern region of Thailand. Being a passive surveillance study, data were collected in line with the enrolling hospitals' policies on the admission and treatment of febrile cases resulting in data being collected from inpatient care at PMKH and both inpatient and outpatient care at KPPH.
The inclusion criteria were any samples collected from dengue clinical diagnosis patients from KPPH (pediatric [0-17 years] and internal medicine [ $\geq 18$ years]), and $\mathrm{PMKH}$ (inpatient pediatric [0-21 years]) from 2002 to 2018, and the exclusion criteria were when consent for future usage of data were not obtained from the patients.

The acute and convalescent blood samples from clinically suspected dengue patients from both hospitals were tested 
TABLE 1

Summary of clinical diagnoses by sex and age between KPPH and PMKH

\begin{tabular}{|c|c|c|c|c|c|c|c|c|}
\hline \multirow[b]{3}{*}{ Sex and age } & \multicolumn{4}{|c|}{ KPPH $(n=11,082)$} & \multicolumn{4}{|c|}{ PMKH $(n=1,065)$} \\
\hline & \multicolumn{3}{|c|}{ Diagnosis count (percent of KPPH total) } & \multirow[b]{2}{*}{ Total febrile cases } & \multicolumn{3}{|c|}{ Diagnosis count (percent of PMKH total) } & \multirow[b]{2}{*}{ Total febrile cases } \\
\hline & DF & DHF & DSS & & DF & DHF & DSS & \\
\hline Female & $3,562(32.1)$ & 1,993 (18.0) & $32(0.3)$ & $5,587(50.4)$ & 395 (37.2) & $49(4.6)$ & $26(2.4)$ & $470(44.2)$ \\
\hline$\leq 2$ & $104(0.9)$ & $38(0.3)$ & - & $142(1.3)$ & $20(1.9)$ & $2(0.2)$ & $1(0.1)$ & $23(2.2)$ \\
\hline $3-10$ & $826(7.5)$ & $353(3.2)$ & $5(0.0)$ & $1,184(10.7)$ & $100(9.4)$ & $15(1.4)$ & $9(0.8)$ & $124(11.6)$ \\
\hline $11-17$ & $1,364(12.3)$ & $785(7.1)$ & $9(0.1)$ & $2,158(19.5)$ & $270(25.4)$ & $30(2.8)$ & $15(1.4)$ & $315(29.6)$ \\
\hline $18-60$ & $1,168(10.5)$ & $775(7.0)$ & $17(0.2)$ & $1,960(17.7)$ & $5(0.5)$ & $2(0.2)$ & $1(0.1)$ & $8(0.8)$ \\
\hline $60+$ & $100(0.9)$ & $42(0.4)$ & $1(0.0)$ & $143(1.3)$ & - & - & - & - \\
\hline Male & 3,607 (32.5) & $1850(16.7)$ & $38(0.3)$ & 5,495 (49.6) & $519(48.7)$ & $45(4.2)$ & $31(2.9)$ & $595(55.8)$ \\
\hline$\leq 2$ & $145(1.3)$ & $45(0.4)$ & - & $190(1.7)$ & $14(1.3)$ & $3(0.3)$ & - & $17(1.6)$ \\
\hline $3-10$ & $798(7.2)$ & $329(3.0)$ & $15(0.1)$ & $1,142(10.3)$ & $108(10.1)$ & $10(0.9)$ & $11(1.0)$ & $129(12.1)$ \\
\hline $11-17$ & $1,497(13.5)$ & $852(7.7)$ & $13(0.1)$ & $2,362(21.3)$ & $390(36.6)$ & $32(3.0)$ & $20(1.9)$ & $442(41.5)$ \\
\hline $18-60$ & $1,100(9.9)$ & $591(5.3)$ & $8(0.1)$ & 1,699 (15.3) & $7(0.7)$ & - & - & $7(0.7)$ \\
\hline $60+$ & $67(0.6)$ & $33(0.3)$ & $2(0.0)$ & $102(0.9)$ & - & - & - & - \\
\hline Total & 7,169 (64.6) & $3,843(34.7)$ & $70(0.7)$ & 11,082 & 914 (85.8) & $94(8.8)$ & $57(5.4)$ & 1,065 \\
\hline
\end{tabular}

for evidence of DENV infection at AFRIMS. Clinical classification was based on WHO guidelines applicable at the time of hospitalization by the clinician. The 1997 WHO dengue classification guidelines were used in this study because the Thailand Ministry of Public Health still reports cases annually using this scheme. ${ }^{12}$ Acute and convalescent blood samples were tested by dengue serological assays, including antiDENV IgM/lgG enzyme immunoassay (EIA) and/or hemagglutination inhibition assay, to differentiate primary infection (the first DENV infection in an individual) and secondary infection (any subsequent DENV infection after primary infection). ${ }^{9,13-16}$ Because of enrolled patients being released

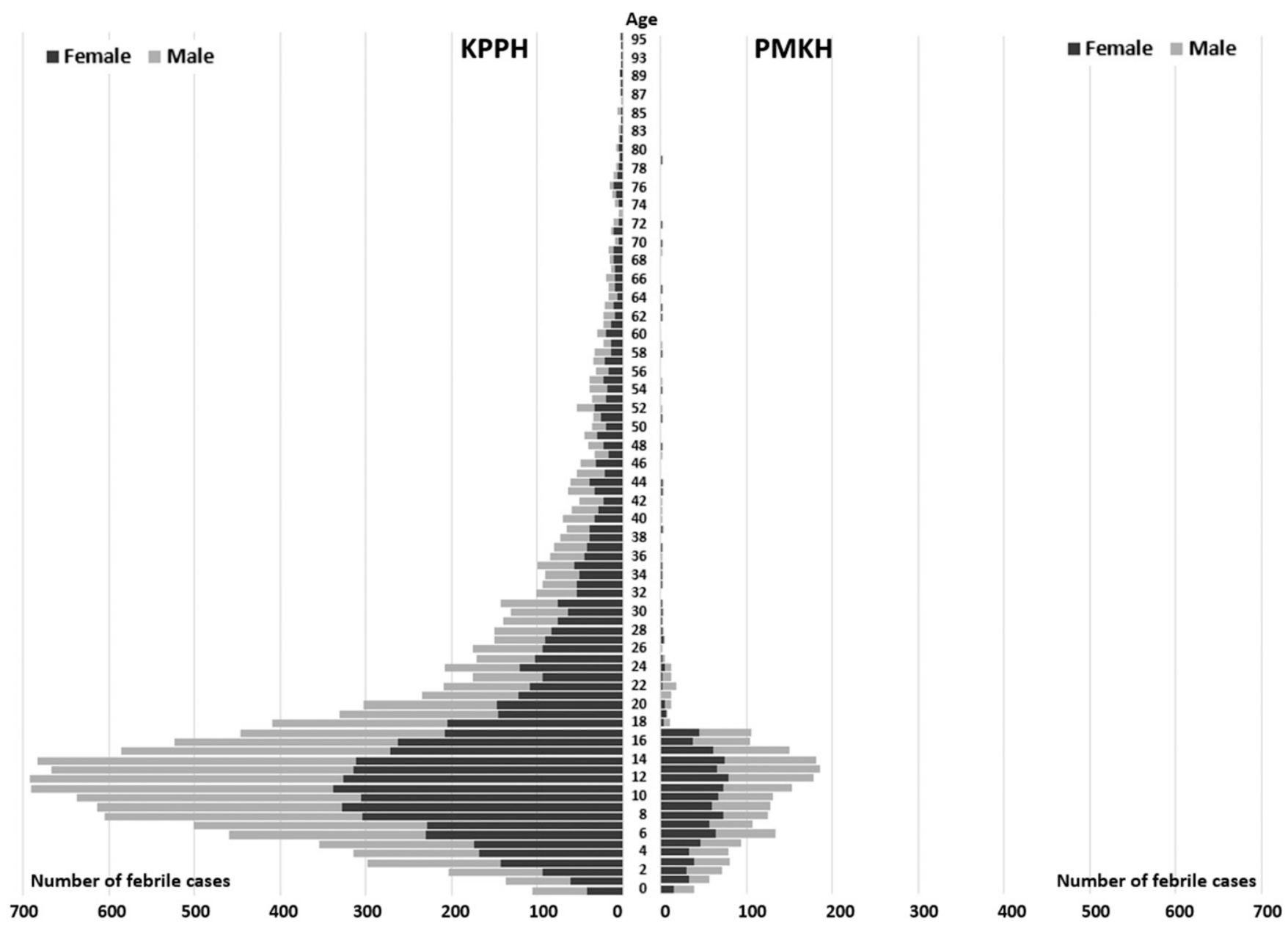

FIGURE 2. Population pyramid summary of patient age data at Kamphaeng Phet Provincial Hospital (KPPH) and Phramongkutklao Hospital (PMKH). 
before collection of an acute and/or convalescent sample or poor subject compliance, six samples were not tested using serological assays from patients at PMKH and 573 samples from KPPH. In addition, a randomly selected subset of serologically interpreted acute blood samples were tested by nested RT-PCR and/or viral isolation. 9,13,14

Serological definitions. An acute primary DENV infection (APDI) is defined as an infection where the ratio of DENV IgM to IgG EIA units in acute and/or convalescent samples are greater than or equal to 1.8, whereas an acute secondary DENV infection (ASDI) would be less than 1.8. An acute DENV infection (ADI) is defined as a single serum sample whose lgM EIA unit is greater than or equal to $40 .^{15,16}$

Statistical analysis. Descriptive statistics were used to summarize the demographics, the distribution of cases over the time, the differences in clinical diagnoses, DENV serotypes, and the serological findings between two sites.

\section{RESULTS}

Study population by sex, age, and clinical diagnosis. Of the 11,082 febrile patients that reported to KPPH and received a dengue related clinical diagnosis during the study period, the number of males and females were evenly distributed $(49.6 \%$, and $50.4 \%$, respectively) with patients from the $11-17$ year age-group making up the largest proportion of the KPPH participants included in the study at $40.8 \%$ (Table 1, Figure 2). The age range of the enrolled patient population at KPPH during the study period was from 1 month to 95 years, with a median age of 14 years. With regard to PMKH's 1,065 febrile dengue patients enrolled during the study period, males made up a larger percentage of the cases at $55.8 \%$, with the largest proportion of $\mathrm{PMKH}$ participants included in the study coming from patients in the 11-17 year age-group at $71.1 \%$. The age range of the $\mathrm{PMKH}$ patients reporting during the study period was from 1 month to 76 years with a median age of 11 years.

At KPPH, dengue fever (DF) made up the largest proportion of clinical diagnoses of enrolled males and females at $65.6 \%$ and $63.8 \%$, followed by DHF at $33.7 \%$ and $35.7 \%$, and finally DSS which was $<1 \%$ for both sexes. Specifically, DF and DHF was seen most commonly among the 11-17 year age-group for both males and females enrolled in the study period at $\mathrm{KPPH}$ representing $13.5 \%$ and $12.3 \%$ and $7.7 \%$ and $7.1 \%$ of cases reported, respectively. The DF and DHF diagnoses were least common among males in the $60+$ year age-group at $0.6 \%$ and $1.3 \%$, respectively, whereas among females, DF was the least common among the $60+$ year old age-group at $0.9 \%$ and DHF among the $\leq 2$ year age-group at $0.3 \%$. There were no cases reported of DSS among both genders in the $\leq 2$ years age-group at KPPH, and a cumulative total of three DSS cases, two male and one female, reported from the 60+ year age-group at KPPH (Table 2, Figure 3).

Similar to KPPH, DF made up the largest proportion of PMKH's clinical diagnoses for enrolled males and females at $87.2 \%$ and $84.0 \%$, followed by DHF at $7.5 \%$ and $10.4 \%$, and finally DSS at $5.2 \%$ and 5.5\%, respectively. Like KPPH, DF, and DHF were seen most commonly among the 11-17 years old age-group for both males and females enrolled in the study period at $\mathrm{PMKH}$ representing $36.6 \%$ and $25.4 \%$ and $3.0 \%$ and $2.8 \%$ of cases reported, respectively. No cases of DF, DHF, or DSS were reported among both genders in the 60+ year agegroup at PMKH (Table 2, Figure 3). In addition, at PMKH, 5.4\% of enrolled patients were diagnosed with DSS, more than 7.7 times as many cases than $\mathrm{KPPH}$ at $0.7 \%$.

Distribution of dengue serotypes during the study period. During the study period, there were varying patterns of which DENV serotypes were the predominate virus in circulation based on location. For KPPH, from 2002 to 2005, a mixed population of four DENV serotypes was observed, with the number of cases between each DENV serotypes not exceeding 100. From 2006 to 2008, there was an emergence of DENV-1 with 116, 211, and 279 cases, respectively (Figure 4). In 2009 , there was a shift to DENV-2 with 97 cases, followed by 152 in 2010, 262 in 2011, and 240 in 2012. Beginning in 2013, DENV-3 emerged and was the predominant serotype among the study participants with 381 cases, followed by 433 cases

TABLE 2

Summary of serological interpretations with respect to age-group and clinical diagnoses among febrile cases

\begin{tabular}{|c|c|c|c|c|c|c|c|c|c|c|c|}
\hline \multirow[b]{3}{*}{ Age-group } & \multirow{3}{*}{$\begin{array}{c}\text { Clinical } \\
\text { diagnosis }\end{array}$} & \multicolumn{4}{|c|}{$\mathrm{KPPH}(n=10,509)$} & \multirow[b]{3}{*}{ Total } & \multicolumn{4}{|c|}{$\mathrm{PMKH}(n=1,059)$} & \multirow[b]{3}{*}{ Total } \\
\hline & & \multicolumn{4}{|c|}{ Serological interpretation (percent of KPPH total) } & & \multicolumn{4}{|c|}{ Serological interpretation (percent of PMKH total) } & \\
\hline & & APDI & ASDI & $A D I$ & NEG & & APDI & ASDI & ADI & NEG & \\
\hline \multirow[t]{3}{*}{$\leq 2$} & DF & $23(0.2)$ & $52(0.5)$ & $14(0.1)$ & $147(1.4)$ & $236(2.2)$ & $6(0.6)$ & $11(1.0)$ & $5(0.5)$ & $12(1.1)$ & 34 (3.2) \\
\hline & $\mathrm{DHF}$ & $26(0.2)$ & $35(0.3)$ & $6(0.1)$ & $16(0.2)$ & $83(0.8)$ & $1(0.1)$ & $3(0.3)$ & - & $1(0.1)$ & $5(0.5)$ \\
\hline & DSS & - & - & - & - & - & $1(0.1)$ & - & - & - & $1(0.1)$ \\
\hline \multirow[t]{3}{*}{$3-10$} & DF & $152(1.4)$ & $903(8.6)$ & $54(0.5)$ & $429(4.1)$ & $1,538(14.6)$ & $22(2.1)$ & $123(11.6)$ & $19(1.8)$ & $40(3.8)$ & 204 (19.3) \\
\hline & DHF & $51(0.5)$ & $520(4.9)$ & $9(0.1)$ & $87(0.8)$ & $667(6.3)$ & $1(0.1)$ & $23(2.2)$ & - & $1(0.1)$ & $25(2.4)$ \\
\hline & DSS & $1(0.0)$ & $16(0.2)$ & - & $1(0.0)$ & $18(0.2)$ & - & $18(1.7)$ & - & $1(0.1)$ & $19(1.8)$ \\
\hline \multirow[t]{3}{*}{$11-17$} & DF & $167(1.6)$ & $1,862(17.7)$ & $45(0.4)$ & $592(5.6)$ & $2,666(25.4)$ & $70(6.6)$ & 456 (43.1) & $37(3.5)$ & $96(9.1)$ & 659 (62.2) \\
\hline & $\mathrm{DHF}$ & $51(0.5)$ & 1,309 (12.5) & $19(0.2)$ & $188(1.8)$ & $1,567(14.9)$ & $3(0.3)$ & $50(4.7)$ & $1(0.1)$ & $8(0.8)$ & $62(5.9)$ \\
\hline & DSS & $1(0.0)$ & $17(0.2)$ & - & $2(0.0)$ & $20(0.2)$ & $2(0.2)$ & $29(2.7)$ & $1(0.1)$ & $3(0.3)$ & $35(3.3)$ \\
\hline \multirow[t]{3}{*}{$18-60$} & DF & $27(0.3)$ & 1,389 (13.2) & $29(0.3)$ & $712(6.8)$ & 2,157 (20.5) & - & $11(1.0)$ & - & $1(0.1)$ & $12(1.1)$ \\
\hline & $\mathrm{DHF}$ & $8(0.1)$ & $1,030(9.8)$ & $15(0.1)$ & 241 (2.3) & $1,294(12.3)$ & - & $2(0.2)$ & - & - & $2(0.2)$ \\
\hline & DSS & - & $16(0.2)$ & - & $8(0.1)$ & $24(0.2)$ & - & $1(0.1)$ & - & - & $1(0.1)$ \\
\hline \multirow[t]{3}{*}{$60+$} & DF & $1(0.0)$ & 69 (0.7) & $1(0.0)$ & $94(0.9)$ & $165(1.6)$ & _- & - & - & _- & - \\
\hline & DHF & $3(0.0)$ & $39(0.4)$ & - & $29(0.3)$ & $71(0.7)$ & - & - & - & - & - \\
\hline & DSS & - & $1(0.0)$ & - & $2(0.0)$ & $3(0.0)$ & - & - & - & - & - \\
\hline \multirow[t]{3}{*}{ Total } & DF & $370(3.5)$ & 4,275 (40.7) & $143(1.4)$ & $1,974(18.8)$ & $6,762(64.3)$ & $98(9.3)$ & $601(56.8)$ & $61(5.8)$ & $149(14.1)$ & 909 (85.8) \\
\hline & DHF & 139 (1.3) & $2,933(27.9)$ & $49(0.5)$ & $561(5.3)$ & $3,682(35.0)$ & $5(0.5)$ & $78(7.4)$ & $1(0.1)$ & $10(0.9)$ & $94(8.9)$ \\
\hline & DSS & $2(0.0)$ & $50(0.5)$ & - & $13(0.1)$ & $65(0.6)$ & $3(0.3)$ & $48(4.5)$ & $1(0.1)$ & $4(0.4)$ & $56(5.3)$ \\
\hline
\end{tabular}

$\mathrm{ADI}$ = acute DENV infection; $\mathrm{APDI}$ = an acute primary DENV infection; ASDI = acute secondary DENV infection; DHF = dengue hemorrhagic fever; DSS = dengue shock syndrome; KPPH = Kamphaeng Phet Provincial Hospital; PMKH = Phramongkutklao Hospital. 


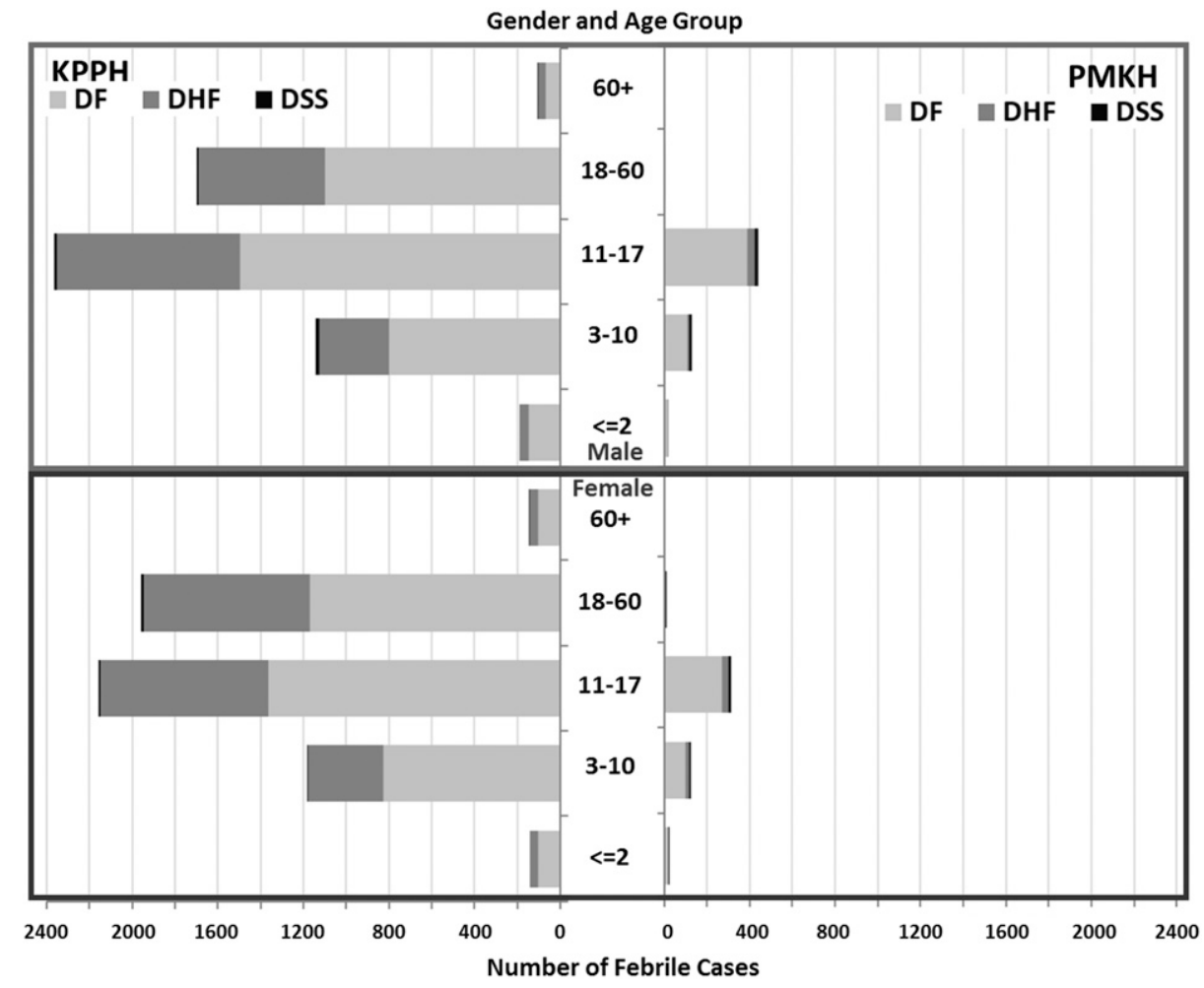

FIGURE 3. Bar chart of clinical diagnoses by sex and age between Kamphaeng Phet Provincial Hospital (KPPH) and Phramongkutklao Hospital (PMKH).

in 2014, and 379 cases in 2015. In 2017, DENV-4 was the predominant serotype isolated with 186 cases, followed by a re-emergence of DENV-1 in 2018 with 388 cases recorded. For PMKH, DENV-1 was the most common serotype among the study participants from 2002 to 2009 (41, 51, 34, 19, 38, 30,51 , and 37 cases, respectively). From 2010 to 2012, there was a mixture of serotypes, all not exceeding 22 cases between each of them. In 2013 and 2015, DENV-3 was the most isolated serotype at 48 and 32 cases, respectively, followed by DENV-2 with 80 cases in 2017, and finally DENV-1 with 61 cases in 2018 (Figure 5).

Serological results by age and study site. To give potential insights into the force of infection of DENV at the sites in the study, an analysis was made to examine the proportion of APDI, ASDI, and ADI with respect to the total number of DENV cases at each site (Table 2, Figure 6). For patients enrolled during the study period at $\mathrm{KPPH}$ and

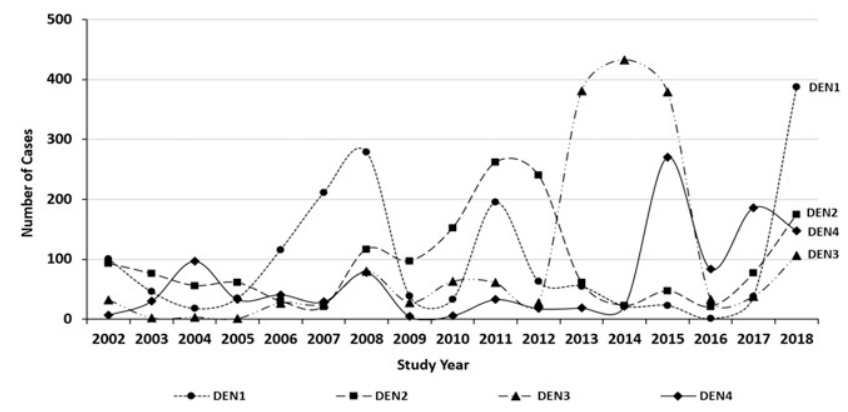

FIGURE 4. Yearly distribution of dengue serotypes between 2002 and 2018 at Kamphaeng Phet Provincial Hospital.
PMKH, DF caused by an ASDI in the 11- to 17-year-old cohort represented the largest portion of cases at $17.7 \%$ and $43.1 \%$, respectively. At $\mathrm{PMKH}$, an ASDI was responsible for $68.7 \%$ of dengue related clinical diagnoses among the enrolled patients in the study, which is 6.8 times as much as the next serological profile seen at PMKH of an APDI at $10.1 \%$. At $\mathrm{KPPH}$, an ASDI was responsible for $69.1 \%$ of dengue related clinical diagnoses among the enrolled patients in the study, which is 14.4 times as much as the next serological profile seen at KPPH of an APDI at $4.8 \%$.

Serological interpretations of clinical diagnosis at each site during the study period. Serological interpretations were made from specimens supplied by both study sites over the 16 years of the study to see if location-dependent trends would emerge. At the KPPH site, a serological interpretation of ASDI accounted for $63.2 \%, 79.6 \%$, and $76.9 \%$ of DF, DHF, and

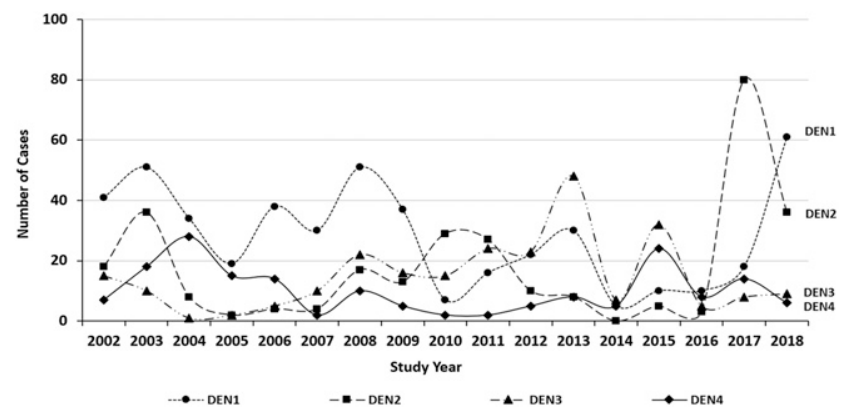

FIGURE 5. Yearly distribution of dengue serotypes between 2002 and 2018 at Phramongkutklao Hospital. 


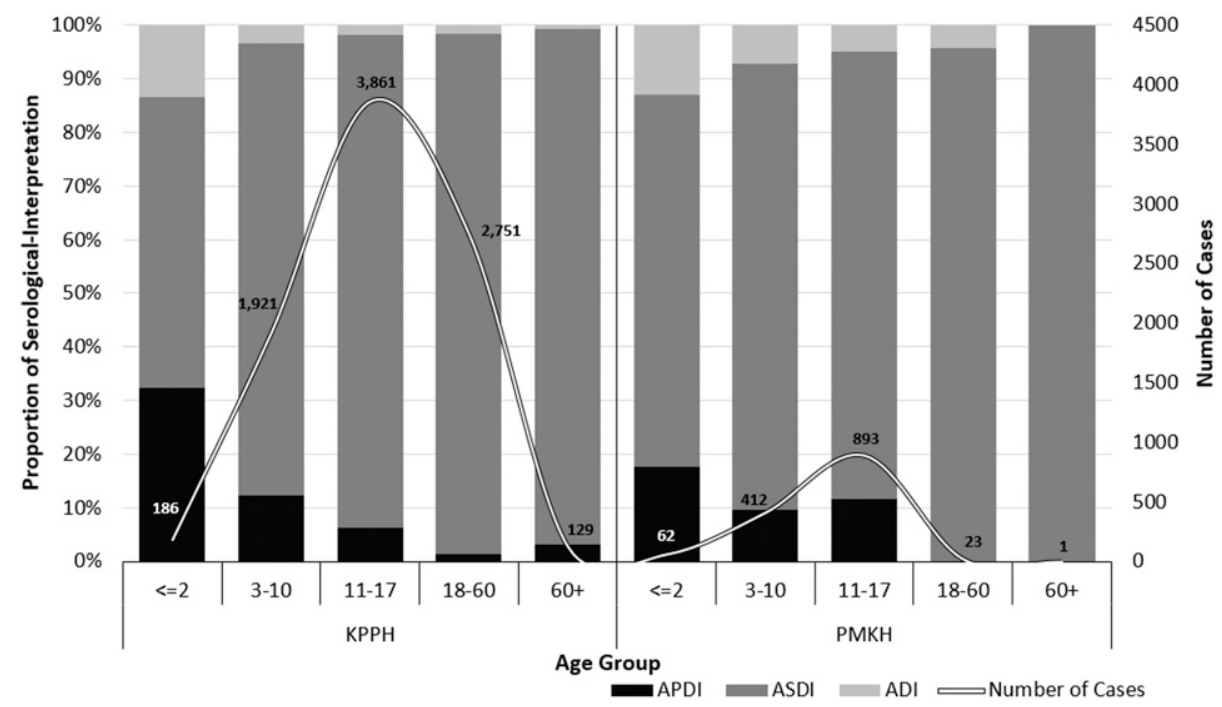

FIGURE 6. Proportion of sero-interpretations with respect to age at Kamphaeng Phet Provincial Hospital (KPPH) and Phramongkutklao Hospital (PMKH).

DSS cases, respectively, as compared with $\mathrm{PMKH}$ at $66.1 \%$, $83.0 \%$, and $85.7 \%$, respectively (Table 2 ).

To determine the impact that DENV specimens from a particular region within Thailand may have on traditional laboratory analysis techniques, the serological interpretations (APDI, ASDI, and ADI) of patients during the study period were compared with RT-PCR results (Table 3). For $\mathrm{KPPH}, 53.1 \%$ of the serologically confirmed DENV cases were positive for RT-PCR, compared with PMKH whose RT-PCR positive specimens made up $82.4 \%$ of total DENV isolates. The majority of positive DENV RT-PCR cases from both KPPH and PMKH were made up of patients with DF as a result of an ASDI, $26.7 \%$ and $54.3 \%$, respectively. The same trend is seen with RT-PCR-negative cases with $27.0 \%$ and $12.9 \%$ of ASDI patients from KPPH and PMKH, respectively, being the largest cohort of negative results (Table 3).

Clinical diagnosis compared with dengue serotyping results. The results of DENV serotyping, using nested RT-PCR, were compared between $\mathrm{KPPH}$ and $\mathrm{PMKH}$ to investigate if there was a difference between subjects who presented with DENV to their respective hospitals (Table 4, Figure 7). For KPPH, DENV-1, DENV-2, DENV-3, and DENV-4 were present across all clinical diagnostic categories at 25.3\%, 26.9\%, 29.0\%, and $18.8 \%$, respectively, with the DENV-3 DF population making up the largest proportion of diagnosed participants at $20.0 \%$. With regard to $\mathrm{PMKH}$, DENV-1 was isolated at $41.1 \%$ across all clinical diagnoses, followed by DENV-3, DENV-2, and DENV-4 at $23.4 \%, 21.5 \%$, and $14.1 \%$, respectively, with the DENV-1 DF cohort making up the largest proportion of diagnosed participants at $34.6 \%$.

\section{DISCUSSION}

All four serotypes of DENV were observed in both hospitals across the study period (2002-2018), although the patterns of emergence varied between the two locations. At PMKH, DENV-1 proved to be the most common, whereas at KPPH, DENV-1, DENV-2, and DENV-3 were predominant at varying points across the study period.

With regard to the age of the patients, at both sites, the 11-17 year age-group provided the greatest proportion of patients that participated in the study period. Interestingly, the second most common age-group varied drastically between the two sites, with KPPH being 18-60, whereas PMKH was $3-10$. This variation could possibly be explained by the collection of samples at both sites where $\mathrm{PMKH}$ received their samples from their pediatric ward, whereas $\mathrm{KPPH}$

TABLE 3

Summary of nested RT-PCR results with respect to serological interpretations at KPPH and PMKH

\begin{tabular}{|c|c|c|c|c|c|c|c|c|c|c|c|c|}
\hline \multirow[b]{3}{*}{ Clinical diagnosis } & \multicolumn{6}{|c|}{$\mathrm{KPPH} n$ (percentage of total KPPH samples) } & \multicolumn{6}{|c|}{ PMKH $n$ (percentage of total PMKH samples) } \\
\hline & \multicolumn{3}{|c|}{ DENV PCR POS } & \multicolumn{3}{|c|}{ DENV PCR NEG } & \multicolumn{3}{|c|}{ DENV PCR POS } & \multicolumn{3}{|c|}{ DENV PCR NEG } \\
\hline & APDI & ASDI & ADI & APDI & ASDI & ADI & APDI & ASDI & ADI & APDI & ASDI & ADI \\
\hline $\mathrm{DF}$ & $268(3.4)$ & $2,129(26.7)$ & $53(0.7)$ & $102(1.3)$ & $2,149(27.0)$ & $91(1.1)$ & $87(9.7)$ & $485(54.3)$ & $51(5.7)$ & $11(1.2)$ & $115(12.9)$ & $10(1.1)$ \\
\hline $\begin{array}{l}\text { Dengue } \\
\text { hemorrhagic } \\
\text { fever }\end{array}$ & $101(1.3)$ & $1,642(20.6)$ & $16(0.2)$ & $39(0.5)$ & $1,295(16.2)$ & $33(0.4)$ & $5(0.6)$ & $64(7.2)$ & - & - & $13(1.5)$ & $1(0.1)$ \\
\hline $\begin{array}{l}\text { Dengue } \\
\text { shock } \\
\text { syndrome }\end{array}$ & $1(0.0)$ & $26(0.3)$ & - & $1(0.0)$ & $24(0.3)$ & - & $2(0.2)$ & $43(4.8)$ & - & $1(0.1)$ & $5(0.6)$ & $1(0.1)$ \\
\hline Total & $370(4.6)$ & $3,797(47.6)$ & $69(0.9)$ & $142(1.8)$ & $3,468(43.5)$ & $124(1.6)$ & $94(10.5)$ & $592(66.2)$ & $51(5.7)$ & $12(1.3)$ & $133(14.9)$ & $12(1.3)$ \\
\hline
\end{tabular}


TABLE 4

Summary of clinical diagnosis compared with DENV nested RT-PCR serotyping results at KPPH and PMKH

\begin{tabular}{|c|c|c|c|c|c|c|c|c|}
\hline \multirow[b]{2}{*}{ Clinical diagnosis } & \multicolumn{4}{|c|}{$\mathrm{KPPH} n$ (percent of total KPPH isolates) } & \multicolumn{4}{|c|}{$\mathrm{PMKH} n$ (percent of total PMKH isolates) } \\
\hline & DEN1 & DEN2 & DEN3 & DEN4 & DEN1 & DEN2 & DEN3 & DEN4 \\
\hline Dengue fever & $822(15.2)$ & $801(14.8)$ & $1,082(20.0)$ & $599(11.1)$ & 308 (34.6) & 159 (17.9) & $185(20.8)$ & $109(12.3)$ \\
\hline Dengue hemorrhagic fever & $533(9.8)$ & 646 (11.9) & $483(8.9)$ & $412(7.6)$ & $30(3.4)$ & $25(2.8)$ & $14(1.6)$ & $10(1.1)$ \\
\hline Dengue shock syndrome & $13(0.2)$ & $8(0.1)$ & $7(0.1)$ & $8(0.1)$ & $27(3.0)$ & $7(0.8)$ & $9(1.0)$ & $6(0.7)$ \\
\hline Total & $1,368(25.3)$ & $1,455(26.9)$ & $1,572(29.0)$ & $1,019(18.8)$ & $365(41.1)$ & $191(21.5)$ & $208(23.4)$ & $125(14.1)$ \\
\hline
\end{tabular}

accessioned samples from both the pediatric and internal medicine wards at their facility.

Analysis of the DENV cases seen at KPPH and PMKH demonstrated that the ASDI serological profile was the most common among participants enrolled in the study at $69.1 \%$ and $68.7 \%$, respectively, with the most common diagnosis, DF, being seen in $66.3 \%$ of all enrolled study participants. Differences, however, were seen when elucidating the serotypes responsible for the clinical diagnoses at the two hospitals. Among DF patient diagnoses, DENV-3 was the most common serotype of study participants seen at KPPH at $20.0 \%$, as opposed to DENV-1 which was the most common serotype of DF study participants seen at PMKH at $34.6 \%$. In cases where DHF was the clinical diagnosis, DENV-2, was the most common serotype of the study participants seen at $\mathrm{KPPH}$ at $11.9 \%$, whereas at $\mathrm{PMKH}$, the most common serotype among DHF study participants was DENV-1 at $3.4 \%$.

Whereas age and gender differences, in the patient population enrolled in the study, were observed between the patients reporting with DENV in the rural areas as compared with those reporting to an urban hospital, the predominant clinical diagnosis and serological profile among all febrile patients enrolled in the study remained DF at $66.3 \%$ and an ASDI at $69.0 \%$, respectively.
Because the study used passive surveillance for the acquisition of the samples, one of the limitations is that the hospitals at different locations and their respective policies for the admission of dengue cases, military medical school hospital at $\mathrm{PMKH}$, and rural provincial government hospital at $\mathrm{KPPH}$ introduced variations in the proportions of dengue illnesses isolated in adults and children between the two sites, making a direct comparison more difficult.

In determining what future efforts need to be studied to elucidate the cause of the difference in serotypes between these two study sites, the effects of climate change on DENV ecology and transmission dynamics should be evaluated such as temperature, rainfall, and humidity. Analysis of Thammapalo et al. ${ }^{17}$ by region showed that DHF incidence was negatively associated with extra rainfall in the southern region but was positively associated with elevated temperatures in the central and northern regions. In addition, Watts et al. ${ }^{18}$ and Kuno et al. ${ }^{19}$ have shown that vector efficiency of Aedes aegypti increases with increasing temperature for DENV allowing for a greater diversity of serotypes to be spread across Thailand. Increasing temperatures also has an impact on viral replication as Rohani et al. ${ }^{20}$ have shown that the time between feeding and virus detection in the salivary glands of Aedes aegypti decreased from 9 days at $26^{\circ} \mathrm{C}$ and $28^{\circ} \mathrm{C}$ to 5 days at $30^{\circ} \mathrm{C}$ for DENV-1 and DENV-4. Thus, examining how these

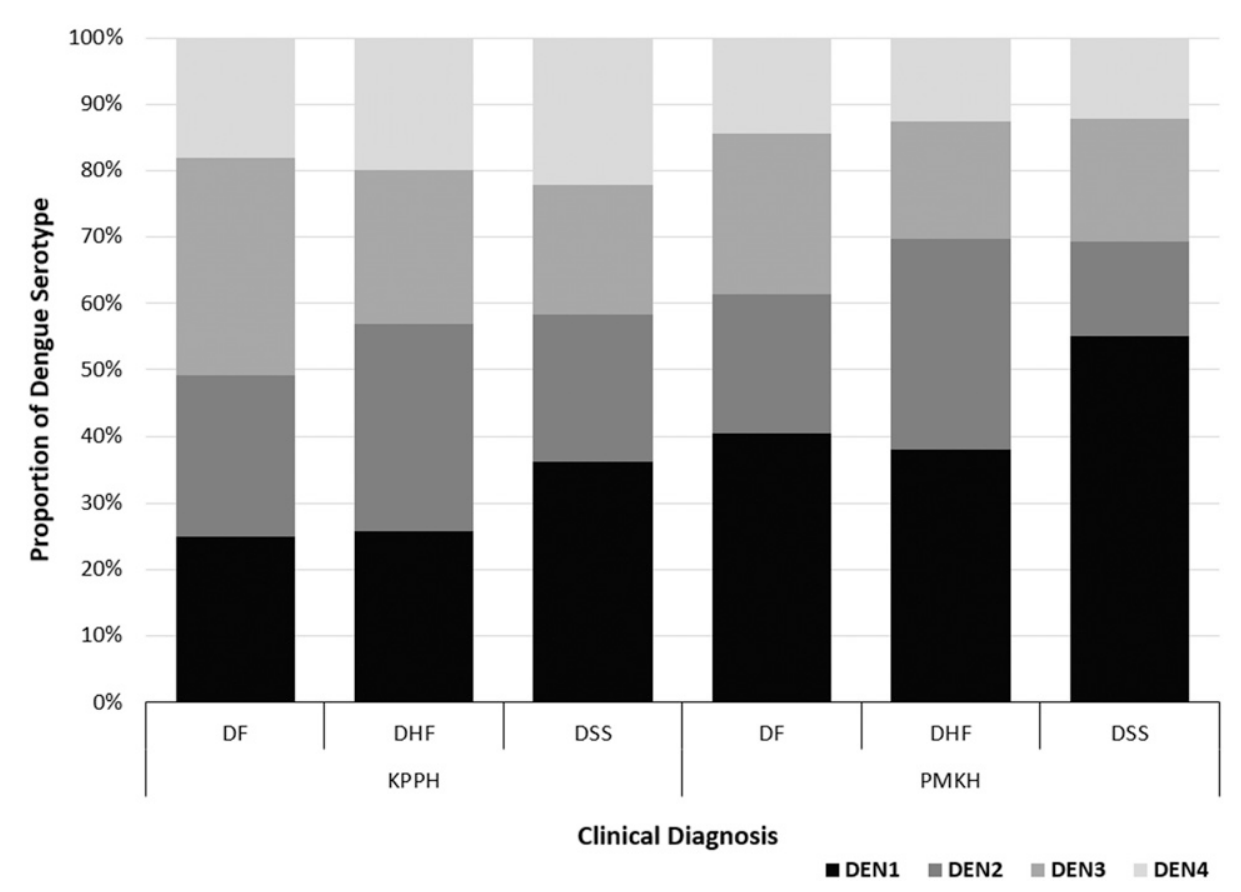

FIGURE 7. Proportion of dengue virus (DENV) serotypes with respect to clinical diagnosis at KPPH and Phramongkutklao Hospital (PMKH). 
serotypes have shifted over time with respect to the changes in temperature over the study period could be explored.

Changes in precipitation can also impact the distribution of dengue serotypes as these events provide habitats for the aquatic stages of the mosquito life cycle and strongly influence vector distribution. ${ }^{21}$

Other weather variables, such as humidity and evaporation rate, influence vector competence, biting behavior, and adult mosquito survival. ${ }^{22}$ For example, in Thailand, ambient temperature appears to define a viable range for transmission, and humidity amplifies the potential within that range. Eighty percent of severe dengue cases over the period 1983-2001 occurred when the temperature was $27-29.5^{\circ} \mathrm{C}$ and mean humidity was $>75 \%$. $^{23}$

In addition, investigations could be made into the migration history of these populations from rural to urban areas and vice versa over the study period. Rabaa et al. ${ }^{24}$ after studying viral lineages in Kamphaeng Phet found more genetic variation across districts in a given year and within the same district across different years. This led the researchers to conclude that the importation of DENV by human movement from other populations is therefore an important generator of DENV diversity even in hyperendemic areas. ${ }^{24}$ In addition, increases in migration, travel, and trade can affect the distribution of vectors and the virus by increasing the density of susceptible individuals. ${ }^{22}$ So tracking the movement of these populations could provide valuable insight into the changes in serotypes that was witnessed.

Finally, examining how the change in demographics over this decade and a half long study could show how the deviation in serotypes could have been established. Traditionally in Thailand, rural households are composed of elderly parents living with their adult children, as opposed to urban areas wherein younger children are living with their parents. These differences in family dynamics could be studied in an attempt to determine the forces of infection for DENV and their specific serotypes. In addition, Cummings et al. ${ }^{25}$ have shown that lower birth and death rates decrease the flow of susceptible individuals into the population and increase the longevity of immune individuals.

These interactions between climatic, socioeconomic, and other factors are complex, vary spatially and temporally and could be further investigated to determine to what degree they influence DENV serotypes throughout Thailand.

Received May 1, 2020. Accepted for publication November 3, 2020.

Published online December 14, 2020.

Acknowledgments: We express our gratitude to all the clinical, laboratory, and support staff at PMKH, KPPH, and AFRIMS.

Financial support: The study was funded by the Armed Forces Health Surveillance Division (AFHSD) Global Emerging Infections Surveillance (GEIS) Branch ProMIS ID: P0081_18_AF_01.02_FY18_FVBI 2018-2020; WRAIR protocol no. 2616.

Disclaimer: Material has been reviewed by the Walter Reed Army Institute of Research. There is no objection to its presentation and/or publication. The opinions or assertions contained herein are the private views of the author and are not to be construed as official, or as reflecting true views of the Department of the Army or the Department of Defense.

Authors' addresses: Phirangkul Kerdpanich and Sriluck Simasathien, Department of Pediatrics, Phramongkutklao Hospital, Bangkok, Thailand, E-mails: akpmkh@yahoo.com and ssriluck@hotmail.com. Suthinee Kongkiatngam, Department of Pediatrics, Kamphaeng Phet
Provincial Hospital (KPPH), Kamphaeng Phet, Thailand, E-mail: suthinee2022@gmail.com. Darunee Buddhari, Kamphaeng Phet-AFRIMS Virology Research Unit, AFRIMS, Kamphaeng Phet, Thailand, E-mail: daruneet.fsn@afrims.org. Chonticha Klungthong, Prinyada Rodpradit, Butsaya Thaisomboonsuk, Tippa Wongstitwilairoong, Taweewun Hunsawong, Stefan Fernandez, and Anthony R. Jones, Department of Virology, Armed Forces Research Institute of Medical Sciences (AFRIMS), Bangkok, Thailand, E-mails: chontichak.fsn@afrims.org, prinyadar.ca@afrims.org, butsayat.fsn@afrims.org, tippaw.ca@afrims.org, taweewunh.fsn@afrims.org, stefan.fernandez.mil@afrims.org, and anthony.jones.mil@afrims.org. Kathryn B. Anderson, Department of Microbiology and Immunology, Upstate Medical University, Syracuse, NY, E-mail: andekath@upstate.edu.

This is an open-access article distributed under the terms of the Creative Commons Attribution (CC-BY) License, which permits unrestricted use, distribution, and reproduction in any medium, provided the original author and source are credited.

\section{REFERENCES}

1. Brady OJ, Gething PW, Bhatt S, Messina JP, Brownstein JS, Hoen AG, Moyes CL, Farlow AW, Scott TW, Hay SI, 2012. Refining the global spatial limits of dengue virus transmission by evidencebased consensus. PLoS Negl Trop Dis 6: e1760.

2. Shepard DS, Undurraga EA, Halasa YA, Stanaway JD, 2016. The global economic burden of dengue: a systematic analysis. Lancet Infect Dis 16: 935-941.

3. Kriengsak L, Brett J, L'Azou M, 2014. Epidemiological trends of dengue disease in Thailand (2000-2011): a systematic literature review. PLoS Negl Trop Dis 8: e3241.

4. Endy TP, Anderson KB, Nisalak A, Yoon IK, Green S, Rothman AL, Thomas SJ, Jarman RG, Libraty DH, Gibbons RV, 2011. Determinants of inapparent and symptomatic dengue infection in a prospective study of primary school children in Kamphaeng Phet, Thailand. PLoS Negl Trop Dis 5: e975.

5. Mammen MP et al., 2008. Spatial and temporal clustering of dengue virus transmission in Thai villages. PLoS Med 5: 1605-1616.

6. Yoon IK et al., 2012. Fine scale spatiotemporal clustering of dengue virus transmission in children and Aedes aegypti in rural Thai villages. PLoS Negl Trop Dis 6: e1730.

7. Yoon IK et al., 2012. Underrecognized mildly symptomatic viremic dengue virus infections in rural Thai schools and villages. $J$ Infect Dis 206: 389-398.

8. Endy TP, Nisalak A, Chunsuttitwat S, Vaughn DW, Green S, Ennis FA, Rothman AL, Libraty DH, 2004. Relationship of preexisting dengue virus (DV) neutralizing antibody levels to viremia and severity of disease in a prospective cohort study of DV infection in Thailand. J Infect Dis 189: 990-1000.

9. Nisalak A, Endy TP, Nimmannitya S, Kalayanarooj S, Thisayakorn U, Scott RM, Burke DS, Hoke CH, Innis BL, Vaughn DW, 2003. Serotype-specific dengue virus circulation and dengue disease in Bangkok, Thailand from 1973 to 1999. Am J Trop Med Hyg 68: 191-202.

10. Kongsomboon $\mathrm{K}$, Singhasivanon $\mathrm{P}$, Kaewkungwal J, Nimmannitya S, Mammen MP, Jr., Nisalak A, Sawanpanyalert P, 2004. Temporal trends of dengue fever/dengue hemorrhagic fever in Bangkok, Thailand from 1981 to 2000: an age-period-cohort analysis. Southeast Asian J Trop Med Public Health 35: 913-917.

11. Fried JR, Gibbons RV, Kalayanarooj S, Thomas SJ, Srikiatkhachorn A, Yoon IK, Jarman RG, Green S, Rothman AL, Cummings DA, 2010. Serotype-specific differences in the risk of dengue hemorrhagic fever: an analysis of data collected in Bangkok, Thailand from 1994 to 2006. PLoS Negl Trop Dis 4: e617.

12. World Health Organization, 1997. Dengue Haemorrhagic Fever: Diagnosis, Treatment, Prevention, and Control. Geneva, Switzerland: WHO, 12-22.

13. Endy TP, Nisalak A, Chunsuttiwat S, Libraty DH, Green S, Rothman AL, Vaughn DW, Ennis FA, 2002. Spatial and temporal circulation of dengue virus serotypes: a prospective study of primary school children in Kamphaeng Phet, Thailand. Am J Epidemiol 156: 52-59. 
14. Endy TP, Yoon IK, Mammen MP, 2010. Prospective cohort studies of dengue viral transmission and severity of disease. Curr Top Microbiol Immunol 338: 1-13.

15. Innis BL, Nisalak A, Nimmannitya S, Kusalerdchariya S, Chongswasdi V, Suntayakorn S, Puttisri P, Hoke CH, 1989. An enzyme-linked immunosorbent assay to characterize dengue infections where dengue and Japanese encephalitis co-circulate. Am J Trop Med Hyg 40: 418-427.

16. Nisalak $A, 2015$. Laboratory diagnosis of dengue virus infections. Southeast Asian J Trop Med Public Health 46: 55-76.

17. Thammapalo S, Chongsuwiwatwong V, McNeil D, Geater A, 2005. The climatic factors influencing the occurrence of dengue hemorrhagic fever in Thailand. Southeast Asian J Trop Med Public Health 36: 191-196.

18. Watts DM, Burke DS, Harrison BA, Whitmire RE, Nisalak A, 1987. Effect of temperature on the vector efficiency of Aedes aegypti for dengue 2 virus. Am J Trop Med Hyg 36: 143152.

19. Kuno G, 1995. Review of the factors modulating dengue transmission. Epidemiol Rev 17: 321-335.
20. Rohani A, Wong YC, Zamre I, Lee HL, Zurainee MN, 2009. The effect of extrinsic incubation temperature on development of dengue serotype 2 and 4 viruses in Aedes aegypti (L.). Southeast Asian J Trop Med Public Health 40: 942-950.

21. Morin CW, Comrie AC, Ernst K, 2013. Climate and dengue transmission: evidence and implications. Environ Health Perspect 121: 1264-1272.

22. Ebi KL, Nealon J, 2016. Dengue in a changing climate. Environ Res 151: 115-123.

23. Campbell KM, Lin CD, lamsirithaworn S, Scott TW, 2013. The complex relationship between weather and dengue virus transmission in Thailand. Am J Trop Med Hyg 89: 1066-1080.

24. Rabaa MA et al., 2013. Frequent in-migration and highly focal transmission of dengue viruses among children in Kamphaeng Phet, Thailand. PLoS Negl Trop Dis 7: e1990.

25. Cummings DA, lamsirithaworn $S$, Lessler JT, McDermott $A$, Prasanthong R, Nisalak A, Jarman RG, Burke DS, Gibbons RV, 2009. The impact of the demographic transition on dengue in Thailand: insights from a statistical analysis and mathematical modeling. PLoS Med 6: e1000139. 